\title{
Contrast Ultrasound Imaging of the Aorta Does Not Affect Progression of Atherosclerosis or Cardiovascular Biomarkers in ApoE-/- Mice
}

\author{
Brendon W. Smith, BS, Douglas G. Simpson, PhD, Sandhya Sarwate, MD, Rita J. Miller, \\ DVM, John W. Erdman Jr, PhD, and William D. O'Brien Jr, PhD \\ Bioacoustics Research Laboratory, Department of Electrical and Computer Engineering (B.W.S., \\ S.S., R.J.M., W.D.O.), Division of Nutritional Sciences (B.W.S., J.W.E., W.D.O.), and \\ Departments of Statistics (D.G.S.), Pathology (S.S.), and Food Science and Human Nutrition \\ (J.W.E.), University of Illinois at Urbana-Champaign, Urbana, Illinois USA
}

\section{Abstract}

Objectives-Ultrasound contrast agents (UCAs) enhance cardiovascular ultrasound imaging. Adverse biological effects have occurred after administration of UCAs, and more research is needed for a comprehensive understanding of the risks involved. We used the $\mathrm{ApoE}^{-/-}$mouse model of atherosclerosis to characterize the effects of ultrasound and UCAs on atherosclerosis and plasma biomarkers.

Methods-Male ApoE ${ }^{-/-}$mice ( 8 weeks old; $\mathrm{n}=24$ ) were intravenously infused with a UCA (2 $\times 10^{10}$ Definity microbubbles per hour; Lantheus Medical Imaging, North Billerica, MA) and exposed to 2.8-MHz center frequency ultrasound $(10 \mathrm{~Hz}$ pulse repetition frequency, 1.4 microseconds pulse duration, 2 minutes exposure duration, and 2 sites) at 1 of 3 derated peak rarefactional pressure amplitudes $(0,1.9$, or $3.8 \mathrm{MPa})$, and then consumed either a chow or Western diet for 4 weeks ( $\mathrm{n}=4$ per group). Blood plasma samples were collected before ultrasound exposure and at 2 and 4 weeks after exposure and assayed for total cholesterol and von Willebrand Factor (vWF). A pathologist measured atheroma thickness in formalin-fixed, hematoxylin-eosin-stained transverse aorta sections and scored them for severity of atherosclerosis.

\begin{abstract}
Results-Plasma total cholesterol initially averaged $286 \mathrm{mg} / \mathrm{dL}$ in the Western diet group and increased to $861 \mathrm{mg} / \mathrm{dL}$ after 4 weeks on the diet $(P<.0001)$. Total cholesterol did not increase significantly in the chow diet group. Plasma vWF increased after 2 weeks on the Western $\operatorname{diet}(P$ $<.0001)$. Atheroma thickness was greater in animals consuming the Western diet than in chow-fed animals $(P<.05)$. Ultrasound had no significant effect on plasma total cholesterol, plasma vWF, or atheroma thickness.
\end{abstract}

Conclusions-Contrast ultrasound did not increase the severity of atherosclerosis or alter cardiovascular biomarkers in the $\mathrm{ApoE}^{-/-}$mouse model. 


\section{Keywords}

atherosclerosis; biomarkers; cardiovascular disease; contrast agents; endothelium; microbubbles; ultrasound

Ultrasound contrast agents (UCAs) are used to enhance ultrasound imaging of the cardiovascular system. Ultrasound contrast agents are administered intravenously as a solution of micrometer-sized bubbles, consisting of an inert gas encased in a shell of phospholipid or albumin. When ultrasound is applied to the heart or blood vessels, the ultrasonic waves interact with UCAs in the circulation, causing the bubbles to oscillate or collapse. The interaction of ultrasound with UCAs opacifies the blood, allowing for improved imaging of cardiac structure and function, ${ }^{1}$ arterial stenosis, ${ }^{2}$ vascularization of atherosclerotic plaques, ${ }^{3}$ and other aspects of the cardiovascular system. When used for cardiac left ventricular opacification, UCAs can enhance the visual quality of ultrasound scans in patients who are difficult to image and improve diagnostic accuracy. 4,5

While UCAs have clear clinical utility in detection and diagnosis of cardiovascular disease, concerns have been raised over their safety. There have been reports of adverse cardiovascular events after ultrasound with UCA administration in animal models and humans. In experimental studies from our research group and others, UCAs have been shown to induce cardiac arrhythmias ${ }^{6}$ and hemorrhage, ${ }^{7-9}$ impair endothelium-dependent vasodilatation, ${ }^{10}$ and alter atheroma thickness and the circulating biomarker von Willebrand Factor (vWF). ${ }^{11}$ Reports of cardiopulmonary complications and deaths in human clinical patients associated with UCA administration added to these concerns, and patients with preexisting cardiovascular disease appeared to be at increased risk. ${ }^{12}$ As a result, the US Food and Drug Administration (FDA) mandated warning labels for 2 commercially available UCAs, Definity (Lantheus Medical Imaging, North Billerica, MA) and Optison (GE Healthcare, Princeton, NJ) in 2007.

The reported adverse events highlight the importance of further research in this area. The biological effects of contrast ultrasound may be a function of the ultrasound pressure level or UCA concentration, so it is necessary to test for adverse events using a variety of scenarios and experimental models. In this study, we used the $\mathrm{ApoE}^{-/-}$mouse model of atherosclerosis to determine thresholds for arterial damage at several different ultrasound pressure levels, with concomitant UCA administration. We fed groups of mice either a standard chow diet or a high-fat, high-sugar, cholesterol-containing Western diet to modulate the progression of atherosclerosis after a single contrast ultrasound exposure. We hypothesized that contrast ultrasound would induce vascular injury and accelerate the progression of atherosclerosis, that this effect would be pressure dependent, and that the threshold for effects of contrast ultrasound on biomarkers would be lower than FDA limits established for clinical ultrasound imaging. 


\section{Materials and Methods}

\section{Animals and Experimental Design}

The University of Illinois Institutional Animal Care and Use Committee approved all live animal procedures. Male $\mathrm{ApoE}^{-/-}$mice ( 8 weeks old, fed chow before study diets; $\mathrm{n}=24$; The Jackson Laboratory, Bar Harbor, ME) were randomized to experimental groups and exposed to ultrasound at 1 of 3 pressure levels with intravenous infusion of a UCA as described in "Exposimetry," with $\mathrm{n}=8$ mice per group. The mice then consumed either a chow diet (7012; Harlan Teklad, Madison, WI) with $3.1 \mathrm{kcal} / \mathrm{g}$, or a Western diet containing $21 \%$ milk fat, $34 \%$ sucrose, $0.2 \%$ (wt/wt) cholesterol, and $4.7 \mathrm{kcal} / \mathrm{g}$ (TD.10885; Harlan Teklad), for 4 weeks after ultrasound exposure ( $\mathrm{n}=4$ per group). Mice were individually housed, fed ad libitum with fresh diet provided twice per week, and given ad libitum access to water. Serial blood samples were collected at baseline, 2 weeks after exposure, and 4 weeks after exposure. Blood samples were collected from the submandibular region by puncture with a 5-mm lancet (Goldenrod; MEDIpoint, Inc, Mineola, NY) into an EDTAcoated capillary tube (Microvette; Sarstedt AG \& Co, Nümbrecht, Germany), centrifuged at $2500 \mathrm{~g}$ and $4^{\circ} \mathrm{C}$ for 10 minutes, aliquoted, and frozen at $-70^{\circ} \mathrm{C}$. Mice were euthanized 4 weeks after exposure, and tissues were collected for analysis (Figure 1).

All mice were anesthetized with isoflurane and euthanized by cervical dislocation. After euthanasia, mice were placed in dorsal recumbency, and a ventral midline incision was made to expose the abdominal and thoracic cavities. The aorta was perfused with $0.8 \mathrm{~mL}$ of $10 \%$ neutral-buffered formalin (NBF) through the left ventricle of the heart and allowed to fix for 30 minutes. An additional $0.2 \mathrm{~mL}$ of NBF was added to the outer surface of the aorta. Next, under magnification, microscissors and fine-point forceps were used to remove the fat surrounding the 3 aortic arch branches (brachiocephalic, left common carotid, and left subclavian). The length of the aorta was removed from its origin at the left ventricle to a few millimeters distal to its bifurcation into the common iliac arteries. The aorta was freed from surrounding vessels by making cuts at each of the 3 branches of the arch, the origin of the aorta at the heart, and distal to the aortic bifurcation into the common iliac arteries.

After removal, the entire aorta was sectioned transversely. The first 2 cuts were made midway between the first and second and second and third aortic branches off the arch. The third cut was made $2 \mathrm{~mm}$ distal to the second cut. All subsequent sections were made 2.5 $\mathrm{mm}$ from the previous section. The average length of aorta removed was $3.5 \mathrm{~cm}$, with 15 sections per aorta. All sections were placed in a single embedding cassette with the distal cut side down in order from the cranial to the caudal ends of the aorta, fixed in 10\% NBF for 24 hours, and embedded in paraffin.

\section{Exposimetry}

Ultrasound exposures were performed with a focused f/2, 19-mm-diameter lithium niobate single-element transducer (Valpey Fisher, Hopkinton, MA) driven by a high-power pulse source (RAM5000; Ritec, Inc, Warwick, RI). Ultrasonic field distribution measurements were performed in $22^{\circ} \mathrm{C}$ degassed water according to established procedures ${ }^{13,14}$ and yielded a center frequency of $2.8 \mathrm{MHz}$, fractional bandwidth of $11 \%$, focal length of $26 \mathrm{~mm}$, 
$-6 \mathrm{~dB}$ focal beam width of $0.98 \mathrm{~mm}$, and $-6 \mathrm{~dB}$ depth of focus of $11 \mathrm{~mm}$. Weekly calibrations were performed as previously described. ${ }^{11}$ A custom transducer holder was machined from aluminum and designed to incorporate both a high-frequency array transducer (MS-400; VisualSonics, Inc, Toronto, Canada) for image guidance and the single-element transducer described above for exposures. The transducers were angled to focus on the same anatomic location at a depth of $14 \mathrm{~mm}$ inside the animal.

Male $\mathrm{ApoE}^{-/-}$mice ( 8 weeks old; $\mathrm{n}=24$ ) were anesthetized with $5 \%$ isoflurane and supplemental oxygen at $2 \mathrm{~L} / \mathrm{min}$ for induction of anesthesia and then maintained at $2 \%$ isoflurane (adjusting the isoflurane level as necessary to keep the animal properly anesthetized) with oxygen at $2 \mathrm{~L} / \mathrm{min}$ via a face mask. Heart and respiratory rates were monitored throughout the experiment. The average heart rate was 368 beats per minute, and the average respiratory rate was 29 breaths per minute. Hair was removed from the ventral side of the animal with clippers (Wahl, Sterling, IL) followed by a depilatory agent (Veet; Reckitt Benckiser, Parsippany, NJ). A high-frequency ultrasound system (Vevo 2100; VisualSonics, Inc, Toronto, Ontario, Canada) was used to image the aorta and position the single-element transducer for ultrasound exposures. Once transducers were positioned, mice were intravenously infused with the UCA $\left(2.6 \%\right.$ Definity in saline, $1.8 \mathrm{~mL} / \mathrm{h}, 2 \times 10^{10}$ microbubbles per hour, and 10 minutes total infusion time) through a lateral tail vein with a 27-gauge $\times 0.5$-in butterfly catheter (Surflo; Terumo, Somerset, NJ) attached to a 1-mL syringe (BD, Franklin Lakes, NJ) and an infusion pump (KD Scientific, Inc, Holliston, MA). When the UCA infusion was started, the aorta was exposed to 2.8-MHz center frequency ultrasound $(10 \mathrm{~Hz}$ pulse repetition frequency, 1.4 microseconds pulse duration, and 2 minutes exposure duration) at 1 of 3 derated peak rarefactional pressure amplitudes (PRPAs; 0, 1.9, or 3.8 MPa). The 1.9-MPa PRPA matched Definity's 90\% collapse threshold. ${ }^{15}$ The mechanical index was also determined as part of the routine calibration procedure ${ }^{16}$ and yielded $0,1.1$, and 2.3 , respectively. The purpose for providing the mechanical index is because it is a regulated quantity ${ }^{17}$ of diagnostic ultrasound systems, and its magnitude is available to system operators. Ultrasound exposures were performed at 2 sites on the aorta: the arch near the branch to the left common carotid artery and the abdominal region immediately caudal to the diaphragm. After the second exposure, the UCA infusion was stopped, and mice were allowed to recover from anesthesia.

\section{Histologic Analysis}

Aorta sections were cut from paraffin blocks at 3- $\mu \mathrm{m}$ thickness, mounted on microscope slides, and stained with hematoxylin-eosin. One microscope slide, with 3 sections of the paraffin block, was created for each animal. A board-certified pathologist blinded to the exposure conditions measured the thickest atheroma in the aorta using an ocular micrometer (Olympus America, Inc, Center Valley, PA) and assigned an atherosclerosis score between 0 and 5 using the American Heart Association classification scheme for human atherosclerotic lesions ${ }^{18}$ : score $0=$ absence of atherosclerosis; score $1=$ presence of isolated foam cells; score 2 = lipid accumulation mainly within the foam cells; score 3 = lipid accumulation within the foam cells and small pools of extracellular lipid; score $4=$ intracellular lipid, lipid pools, and core of extracellular lipid; and score $5=$ lipid core and fibrotic layer, multiple lipid cores and fibrotic layer, or mainly calcified or fibrotic plaque. 


\section{Blood Analysis}

Plasma total cholesterol was measured using an enzymatic colorimetric kit (Wako Chemicals, Richmond, VA) with samples plated in triplicate. Human control sera (Wako) were included in each assay to evaluate the reproducibility of measurement. Intra-assay precision was defined as the variability among replicates of the same sample within an assay and inter-assay precision as the variability among replicates of the same sample over multiple assays. A coefficient of variation (CV) was used as a measure of precision ${ }^{19}$ and defined as (standard deviation)/mean $\times 100$. The intra-assay $\mathrm{CV}$ for control sera was $3.5 \%$, and the inter-assay CV was $9.2 \%$. The intra-assay CV for mouse plasma samples was $2.3 \%$.

von Willebrand factor was measured using a sandwich enzyme-linked immunosorbent assay as previously described ${ }^{20}$ with the following modifications: to adapt the assay for mouse plasma instead of rabbit plasma, a standard curve from 25 to $0.39 \mathrm{ng} / \mathrm{mL}$ was used instead of 100 to $3.13 \mathrm{ng} / \mathrm{mL}$, and mouse plasma was diluted 1:25 for analysis instead of 1:100.

Samples were plated in duplicate. The same antibodies and reagents were used for mouse and rabbit plasma, as a BLAST search ${ }^{21}$ demonstrates $83 \%$ sequence alignment between human (accession number CCQ25771.1) and mouse (accession number AAN07781.2) vWF and $81 \%$ between mouse and rabbit (accession number DAA34807.1) vWF. For mouse plasma samples, the intra-assay CV was 3.2\%, and the inter-assay CV was $13 \%$.

\section{Statistical Analysis}

Statistical analysis was performed in SAS 9.3 for Windows (SAS Institute, Inc, Cary, NC), and graphs were created in SigmaPlot 12.3 for Windows (Systat Software, Inc, San Jose, CA). Atheroma thickness data were analyzed by analysis of variance within the general linear mixed models procedure of SAS 9.3. Feed intake, body weights, plasma total cholesterol, and plasma vWF data were analyzed by repeated measures analysis of covariance, using baseline values as covariates, also within the general linear mixed models procedure of SAS 9.3. The covariance structure of repeated measures data was assessed graphically and quantitatively, and an appropriate covariance model was fit. Nonparametric atherosclerosis score data were analyzed using a 2-sample Wilcoxon rank sum test with diet as the treatment variable. Each model was first run with all fixed effects and covariates of interest (diet, time point, PRPA, and baseline values) incorporated and then reduced to eliminate nonsignificant variables. When a significant result was reported for a fixed variable with more than 2 categories, multiple comparisons were made using estimate statements based on the least squares means, followed by Bonferroni adjustment for multiplicity. Data met the necessary assumptions of each test. The threshold for statistical significance was set at $P<.05$.

\section{Results}

\section{Contrast Ultrasound Does Not Affect Feed Intake or Body Weight}

Animals on the chow diet consumed more feed by weight but less kilocalories than animals on the Western diet $(4.1 \mathrm{~g} / \mathrm{d}$ feed intake for chow animals versus $3.3 \mathrm{~g} / \mathrm{d}$ for Western diet animals; $P<.0005 ; 12.8 \mathrm{kcal} / \mathrm{d}$ feed intake for chow animals versus $15.4 \mathrm{kcal} / \mathrm{d}$ for Western diet animals; $P<.005)$. Animals on the chow diet weighed less than animals on the Western 
diet (24.6 g final body weight for chow animals versus $26.5 \mathrm{~g}$ for Western diet animals; $P<$. 005). Ultrasound did not significantly affect feed intake or body weight.

\section{Contrast Ultrasound Does Not Affect Plasma Cholesterol or vWF}

Total cholesterol initially averaged $283 \mathrm{mg} / \mathrm{dL}$ and did not increase significantly in the chow diet group (Figure 2A). While cholesterol levels in chow-fed animals remained stable, animals in the Western diet groups had a rapid and significant elevation to an average of 726 $\mathrm{mg} / \mathrm{dL}$ after 2 weeks on the diet $(P<.0001$ by repeated measures analysis of covariance), and levels remained significantly elevated after that, averaging $861 \mathrm{mg} / \mathrm{dL}$ after 4 weeks. Ultrasound $(P=.25)$, diet $\times$ ultrasound $(P=.89)$, and diet $\times$ ultrasound $\times$ time point $(P=$. 37 ) were not significant variables in the statistical model and so were removed.

Plasma vWF initially averaged $112 \mathrm{ng} / \mathrm{mL}$ in all animals (Figure 2B). Consumption of the Western diet for 2 weeks significantly elevated $\mathrm{vWF}$ to an average of $257 \mathrm{ng} / \mathrm{mL}$ compared to $78 \mathrm{ng} / \mathrm{mL}$ for animals continuing to consume chow $(P<.0001$ by repeated measures analysis of covariance). Levels were highly variable at 4 weeks, and no significant differences between diets or ultrasound groups were seen.

\section{Contrast Ultrasound Does Not Affect Aortic Atheroma Thickness or Atherosclerosis Score}

Atheroma thickness was significantly greater in animals consuming the Western diet than in chow-fed animals, with an average \pm SEM of $0.031 \pm 0.010 \mathrm{~mm}$ for chow and $0.073 \pm 0.014$ $\mathrm{mm}$ for Western at $0 \mathrm{MPa}, 0.020 \pm 0.007 \mathrm{~mm}$ for chow and $0.039 \pm 0.012 \mathrm{~mm}$ for Western at $1.9 \mathrm{MPa}$, and $0.025 \pm 0.014 \mathrm{~mm}$ for chow and $0.036 \pm 0.010 \mathrm{~mm}$ for Western at $3.8 \mathrm{MPa}$ $(P<.05$ by analysis of variance; $\mathrm{n}=3-4$ per group; Figure $3 \mathrm{~B})$. Ultrasound $(P=.11)$ and diet $\times$ ultrasound $(P=.40)$ were not significant variables in the statistical model and so were removed. If the diet $\times$ ultrasoundvariable was retained, the ultrasound variable would approach statistical significance within the Western diet group only $(P<.08)$, and there would be a significant difference between Western and chow at 0 PRPA $(P<.05)$, but not $1.9(P=.24)$ or $3.8(P=.50)$ PRPA. A regression analysis of the atheroma thickness data, within either the Western diet group or the chow diet group, and treating both the atheroma thickness and the ultrasound PRPA as continuous variables, did not identify any significant trends among PRPA groups $\left[F_{0.05(1,9)}=2.51 ; r^{2}=0.22 ; P=.15\right.$ for Western; $F_{0.05}(1,9)=$ $0.08 ; r^{2}=0.009 ; P=.78$ for chow).

The atherosclerosis score was also significantly greater for Western diet-fed animals than chow-fed animals, with an average \pm SEM of $2.25 \pm 0.946$ for chow and $3.33 \pm 0.882$ for Western at $0 \mathrm{MPa}, 1.25 \pm 0.479$ for chow and $2.00 \pm 0.408$ for Western at $1.9 \mathrm{MPa}$, and 1.00 \pm 0.577 for chow and $2.50 \pm 0.866$ for Western at $3.8 \mathrm{MPa}$ (1-sided $P<.05$ by Wilcoxon rank sum test; $\mathrm{n}=3-4$ per group; Figure $3 \mathrm{C})$. Ultrasound was not a significant variable overall $(P=.45)$, within the chow diet group alone $(P=.62)$, or within the Western diet group alone $(P=.25)$ for atherosclerosis score.

\section{Discussion}

Concerns over the safety of UCAs began after reports of adverse cardiopulmonary events and deaths following their administration. Based on these reports, the FDA required revised 
labeling for Definity and Optison in 2007, adding a "black box" label warning of adverse events and requiring a 30-minute monitoring period for all patients injected with UCAs. However, a small number of adverse events were reported during a span of several years in which millions of patients were safely imaged, so the estimated incidence of adverse events was far lower than for many established medical procedures. ${ }^{22}$ The few deaths that did occur were likely due to underlying disease conditions rather than UCAs. ${ }^{23}$ A large body of literature has emerged to demonstrate the safety of UCAs, ${ }^{12,24-27}$ and a recent metaanalysis ${ }^{28}$ of more than 5 million patients concluded that the use of UCAs in ultrasound imaging is safe and not associated with an increased risk of myocardial infarction or death.

After these reports of UCA safety accumulated, the FDA relaxed its warnings on Definity in October 2011 and Optison in August 2012, noting that serious complications are uncommon and removing the 30-minute monitoring period after UCA injection. This study adds support for the safety of UCAs and suggests that UCAs can be used even in patients with atherosclerosis.

In addition to their growing safety record, UCAs show promise in therapeutic applications. They have been used to enhance gene and drug delivery, ${ }^{29,30}$ reduce infarct size and improve ventricular remodeling after cardiac ischemia, ${ }^{31}$ and induce angiogenesis to improve tissue perfusion. ${ }^{32-37}$ These innovative functions of UCAs, in conjunction with their safety record, make a strong case for their continued use in the laboratory and clinic.

We selected the $\mathrm{ApoE}^{-/-}$mouse as a model of atherosclerosis for our investigations, a wellestablished mouse model ${ }^{38}$ that recapitulates many aspects of human atherosclerosis. ${ }^{39}$ ApoE is an apolipoprotein important for cholesterol metabolism, particularly in rodents. ${ }^{40}$ Deletion of ApoE results in impaired hepatic clearance of lipoproteins from the circulation. As a result, lipoproteins accumulate in the blood, reaching nearly $900 \mathrm{mg} / \mathrm{dL}$ in this study, and infiltrate arterial tissue to promote atherosclerosis. The $\mathrm{ApoE}^{-/-}$mouse provides several advantages over the cholesterol-fed rabbit model also used by our research group. ${ }^{41,42}$ The mice are easier to handle and readily consume the atherosclerotic diet. As in our previous study, ${ }^{11}$ we measured the biomarker $\mathrm{vWF}$, a protein produced and secreted by vascular endothelial cells. Alterations in plasma vWF levels reflect endothelial dysfunction ${ }^{43}$ and predict the risk of cardiovascular events. ${ }^{44} \mathrm{The}^{\mathrm{ApoE}} \mathrm{F}^{-/}$mouse, validated assays, and histologic techniques comprise a sensitive model system to test for biological effects of contrast ultrasound.

Our findings in this study were different than those observed in the cholesterol-fed rabbit model. ${ }^{11}$ The lack of concordance between the two studies may be due to methodological and/or physiologic differences. First, we are working with a different animal model, and mice may simply respond differently to ultrasound and UCAs than rabbits. The increased heart, respiratory, and metabolic rates of mice may decrease the half-life of UCAs. In this study, we did not collect blood samples immediately before and after exposure. We also measured plaque thickness by examining the entire aortic arch and descending aorta, instead of just the specific ultrasound exposure site, which may explain why we did not observe alterations in aorta plaque thickness. Small sample sizes may also have limited our statistical power. Further studies are needed to determine whether differences in results between the two studies are due to differences in experimental methods or animal physiology. 
In conclusion, contrast ultrasound did not affect clinically relevant cardiovascular biomarkers or progression of atherosclerosis in $\mathrm{ApoE}^{-/-}$mice. This study represents an important step in understanding the biological effects of UCAs and adds to a growing body of literature demonstrating their safety in cardiovascular ultrasound imaging. The focus of this research area has begun to shift away from the potential of UCAs to cause cardiovascular harm and on to improving cardiovascular health and diagnosis, and it has become clear that the benefits of UCAs outweigh the potential risks in almost all current applications.

\section{Acknowledgments}

We thank Emily Hartman, Rami Abuhabsah, Michael Kurowski, Jessica Ang, and Presence Covenant Medical Center for their contributions. This work was supported by National Institutes of Health grant R37EB002641.

\section{Abbreviations}

$\begin{array}{ll}\text { CV } & \text { coefficient of variation } \\ \text { FDA } & \text { Food and Drug Administration } \\ \text { NBF } & \text { neutral-buffered formalin } \\ \text { PRPA } & \text { derated peak rarefactional pressure amplitude } \\ \text { UCA } & \text { ultrasound contrast agent } \\ \text { vWF } & \text { von Willebrand factor }\end{array}$

\section{References}

1. Senior R, Shah BN. Myocardial contrast echocardiography for simultaneous assessment of function and perfusion in real time: a technique comes of age. Circulation. 2012; 126:1182-1184. [PubMed: 22872313]

2. Porter T, Li S, Kilzer K, Deligonul U. Correlation between quantitative angiographic lesion severity and myocardial contrast intensity during a continuous infusion of perfluorocarbon-containing microbubbles. J Am Soc Echocardiogr. 1998; 11:702-710. [PubMed: 9692527]

3. Akkus Z, Hoogi A, Renaud G, et al. New quantification methods for carotid intra-plaque neovascularization using contrast-enhanced ultrasound. Ultrasound Med Biol. 2014; 40:25-36. [PubMed: 24161799]

4. Kitzman DW, Goldman ME, Gillam LD, Cohen JL, Aurigemma GP, Gottdiener JS. Efficacy and safety of the novel ultrasound contrast agent perflutren (Definity) in patients with suboptimal baseline left ventricular echocardiographic images. Am J Cardiol. 2000; 86:669-674. [PubMed: 10980221]

5. Reilly JP, Tunick PA, Timmermans RJ, Stein B, Rosenzweig BP, Kronzon I. Contrast echocardiography clarifies uninterpretable wall motion in intensive care unit patients. J Am Coll Cardiol. 2000; 35:485-490. [PubMed: 10676698]

6. Zachary JF, Hartleben SA, Frizzell LA, O'Brien WD Jr. Arrhythmias in rat hearts exposed to pulsed ultrasound after intravenous injection of a contrast agent. J Ultrasound Med. 2002; 21:1347-1356. [PubMed: 12494976]

7. Miller DL, Quddus J. Diagnostic ultrasound activation of contrast agent gas bodies induces capillary rupture in mice. Proc Natl Acad Sci USA. 2000; 97:10179-10184. [PubMed: 10954753]

8. Miller DL, Dou C, Sorenson D, Liu M. Histological observation of islet hemorrhage induced by diagnostic ultrasound with contrast agent in rat pancreas. PloS One. 2011; 6:e21617. [PubMed: 21738734] 
9. American Institute of Ultrasound in Medicine. Section 6: mechanical bioeffects in the presence of gas-carrier ultrasound contrast agents. J Ultrasound Med. 2000; 19:120-142. 154-168. [PubMed: 10680618]

10. Wood SC, Antony S, Brown RP, et al. Effects of ultrasound and ultrasound contrast agent on vascular tissue. Cardiovasc Ultrasound. 2012; 10:29. [PubMed: 22805356]

11. Smith BW, Simpson DG, Sarwate S, et al. Contrast ultrasound imaging of the aorta alters vascular morphology and circulating von Willebrand factor in hypercholesterolemic rabbits. J Ultrasound Med. 2012; 31:711-720. [PubMed: 22535718]

12. Main ML. Ultrasound contrast agent safety: from anecdote to evidence. JACC Cardiovasc Imaging. 2009; 2:1057-1059. [PubMed: 19761982]

13. Raum K, O’Brien WD Jr. Pulse-echo field distribution measurement technique of high-frequency ultrasound sources. IEEE Trans Ultrason Ferroelectr Freq Control. 1997; 44:810-815.

14. Zachary JF, Sempsrott JM, Frizzell LA, Simpson DG, O’Brien WD Jr. Superthreshold behavior and threshold estimation of ultrasound-induced lung hemorrhage in adult mice and rats. IEEE Trans Ultrason Ferroelectr Freq Control. 2001; 48:581-592. [PubMed: 11370372]

15. King DA, O'Brien WD Jr. Comparison between maximum radial expansion of ultrasound contrast agents and experimental postexcitation signal results. J Acoust Soc Am. 2011; 129:114-121. [PubMed: 21302993]

16. American Institute of Ultrasound in Medicine, National Electrical Manufacturers Association. Standard for Real-time Display of Thermal and Mechanical Acoustic Output Indices on Diagnostic Ultrasound Equipment. Laurel, MD: American Institute of Ultrasound in Medicine; Rosslyn, VA: National Electrical Manufacturers Association; 2004.

17. US Food and Drug Administration, Center for Devices and Radiological Health. Information for Manufacturers Seeking Marketing Clearance of Diagnostic Ultrasound Systems and Transducers. Rockville, MD: US Food and Drug Administration; 1997.

18. Kumar, V.; Fausto, N.; Abbas, AK. Robbins and Cotran Pathologic Basis of Disease. 7. Philadelphia, PA: WB Saunders Co; 2005.

19. Reed GF, Lynn F, Meade BD. Use of coefficient of variation in assessing variability of quantitative assays. Clin Diagn Lab Immunol. 2002; 9:1235-1239. [PubMed: 12414755]

20. Smith BW, Strakova J, King JL, Erdman JW Jr, O’Brien WD Jr. Validated sandwich ELISA for the quantification of von Willebrand factor in rabbit plasma. Biomark Insights. 2010; 5:119-127. [PubMed: 21151589]

21. Altschul SF, Madden TL, Schäffer AA, et al. Gapped BLAST and PSI-BLAST: a new generation of protein database search programs. Nucleic Acids Res. 1997; 25:3389-3402. [PubMed: 9254694]

22. Grayburn PA. Product safety compromises patient safety (an unjustified black box warning on ultrasound contrast agents by the Food and Drug Administration). Am J Cardiol. 2008; 101:892893. [PubMed: 18328861]

23. Main ML, Goldman JH, Grayburn PA. Thinking outside the "box": the ultrasound contrast controversy. J Am Coll Cardiol. 2007; 50:2434-2437. [PubMed: 18154971]

24. Herzog CA. Incidence of adverse events associated with use of perflutren contrast agents for echocardiography. JAMA. 2008; 299:2023-2025. [PubMed: 18460662]

25. Wei K, Mulvagh SL, Carson L, et al. The safety of Definity and Optison for ultrasound image enhancement: a retrospective analysis of 78,383 administered contrast doses. J Am Soc Echocardiogr. 2008; 21:1202-1206. [PubMed: 18848430]

26. Dolan MS, Gala SS, Dodla S, et al. Safety and efficacy of commercially available ultrasound contrast agents for rest and stress echocardiography: a multicenter experience. J Am Coll Cardiol. 2009; 53:32-38. [PubMed: 19118722]

27. Abdelmoneim SS, Bernier M, Scott CG, et al. Safety of contrast agent use during stress echocardiography in patients with elevated right ventricular systolic pressure: a cohort study. Circ Cardiovasc Imaging. 2010; 3:240-248. [PubMed: 20233859]

28. Khawaja OA, Shaikh KA, Al-Mallah MH. Meta-analysis of adverse cardiovascular events associated with echocardiographic contrast agents. Am J Cardiol. 2010; 106:742-747. [PubMed: 20723656] 
29. Bekeredjian R, Grayburn PA, Shohet RV. Use of ultrasound contrast agents for gene or drug delivery in cardiovascular medicine. J Am Coll Cardiol. 2005; 45:329-335. [PubMed: 15680708]

30. Villanueva FS. Getting good vibes: the therapeutic power of microbubbles and ultrasound. JACC Cardiovasc Imaging. 2012; 5:1263-1266. [PubMed: 23236977]

31. Dörner J, Struck R, Zimmer S, et al. Ultrasound-mediated stimulation of microbubbles after acute myocardial infarction and reperfusion ameliorates left-ventricular remodelling in mice via improvement of borderzone vascularization. PloS One. 2013; 8:e56841. [PubMed: 23437254]

32. Song J, Qi M, Kaul S, Price RJ. Stimulation of arteriogenesis in skeletal muscle by microbubble destruction with ultrasound. Circulation. 2002; 106:1550-1555. [PubMed: 12234963]

33. Chappell JC, Klibanov AL, Price RJ. Ultrasound-microbubble-induced neovascularization in mouse skeletal muscle. Ultrasound Med Biol. 2005; 31:1411-1422. [PubMed: 16223645]

34. Chappell JC, Song J, Klibanov AL, Price RJ. Ultrasonic microbubble destruction stimulates therapeutic arteriogenesis via the CD18-dependent recruitment of bone marrow-derived cells. Arterioscler Thromb Vasc Biol. 2008; 28:1117-1122. [PubMed: 18403725]

35. Johnson CA, Sarwate S, Miller RJ, O’Brien WD Jr. A temporal study of ultrasound contrast agentinduced changes in capillary density. J Ultrasound Med. 2010; 29:1267-1275. [PubMed: 20733181]

36. Johnson CA, Miller RJ, O'Brien WD Jr. Ultrasound contrast agents affect the angiogenic response. J Ultrasound Med. 2011; 30:933-941. [PubMed: 21705726]

37. Johnson CA, O'Brien WD Jr. The angiogenic response is dependent on ultrasound contrast agent concentration. Vasc Cell. 2012; 4:10. [PubMed: 22587914]

38. Getz GS, Reardon CA. Diet and murine atherosclerosis. Arterioscler Thromb Vasc Biol. 2006; 26:242-249. [PubMed: 16373607]

39. Smith JD. Mouse models of atherosclerosis. Lab Anim Sci. 1998; 48:573-579. [PubMed: 10090079]

40. Rader DJ, FitzGerald GA. State of the art: atherosclerosis in a limited edition. Nat Med. 1998; 4:899-900. [PubMed: 9701240]

41. King JL, Miller RJ, Blue JP Jr, O’Brien WD Jr, Erdman JW Jr. Inadequate dietary magnesium intake increases atherosclerotic plaque development in rabbits. Nutr Res. 2009; 29:343-349. [PubMed: 19555816]

42. Smith BW, King JL, Miller RJ, et al. Optimization of a low magnesium, cholesterol-containing diet for the development of atherosclerosis in rabbits. J Food Res. 2013; 2:168-178. [PubMed: 24672587]

43. Constans J, Conri C. Circulating markers of endothelial function in cardiovascular disease. Clin Chim Acta. 2006; 368:33-47. [PubMed: 16530177]

44. Spiel AO, Gilbert JC, Jilma B. von Willebrand factor in cardiovascular disease:focus on acute coronary syndromes. Circulation. 2008; 117:1449-1459. [PubMed: 18347221] 


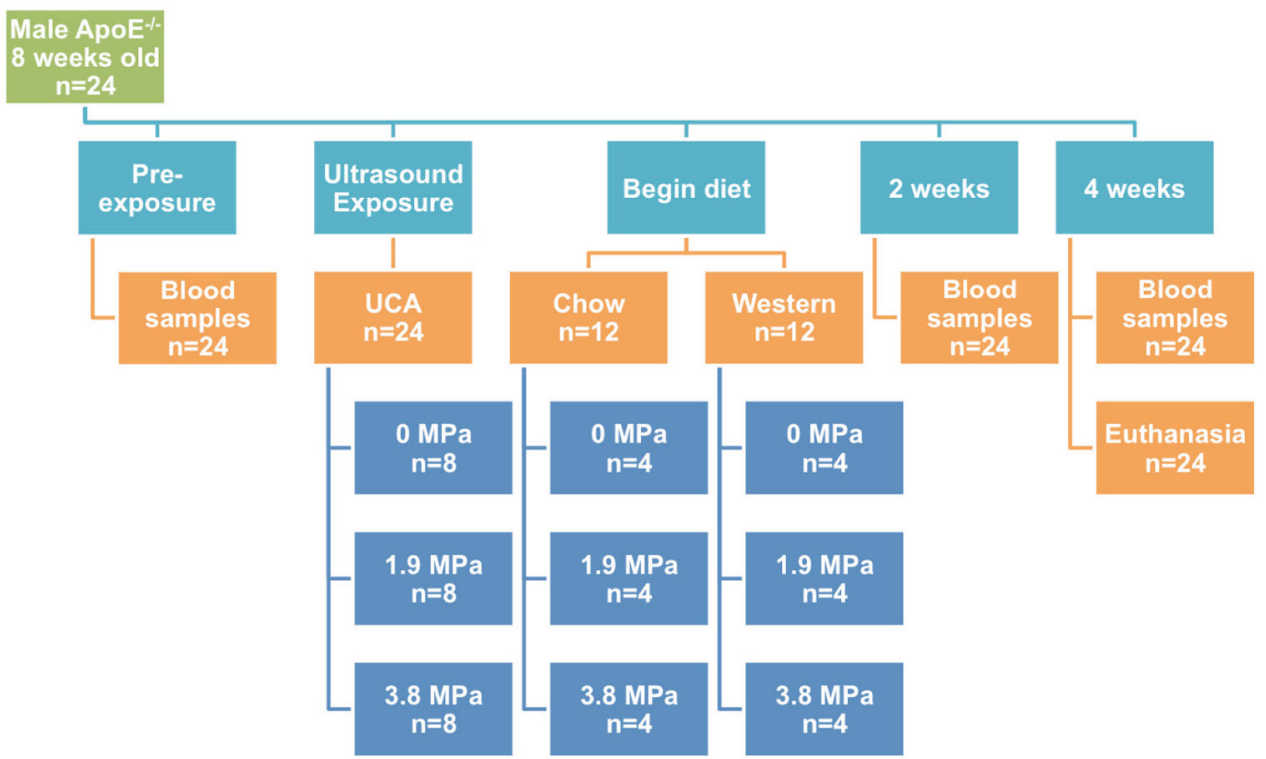

Figure 1.

Study design. Baseline blood samples were obtained from 24 male ApoE-/- mice. The mice then received the UCA and were exposed to ultrasound at $0,1.9$, or $3.8 \mathrm{MPa}$ in situ PRPA, with $\mathrm{n}=8$ per group. Half of the mice continued to consume the chow diet, and half were switched to the Western diet. Subsequent blood samples were obtained at 2 and 4 weeks. Mice were euthanized at 4 weeks, and aortas were obtained for analysis. 


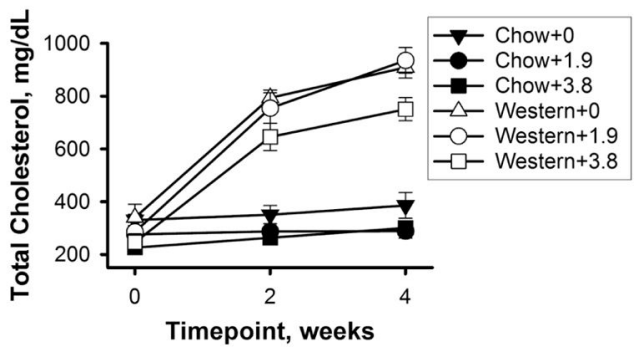

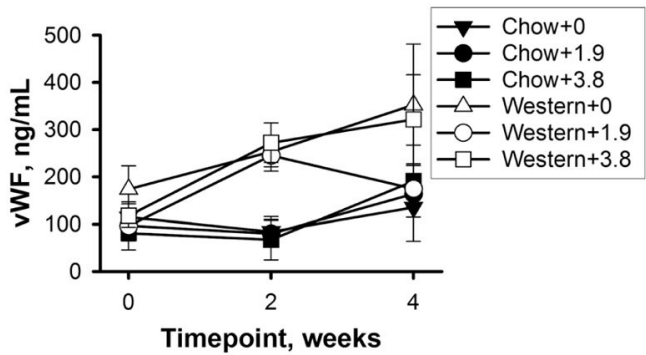

Figure 2.

Plasma cholesterol (A) and vWF (B). Plasma total cholesterol was analyzed using an enzymatic colorimetric kit. Human control sera were included in each assay for quality control. Plasma vWF was determined using an in-house validated sandwich enzyme-linked immunosorbent assay. Bars indicate SEM; $n=3$ or 4 per group per time point. 


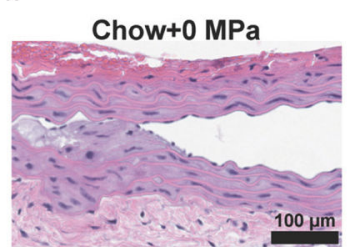

Chow+1.9 MPa Chow+3.8 MPa
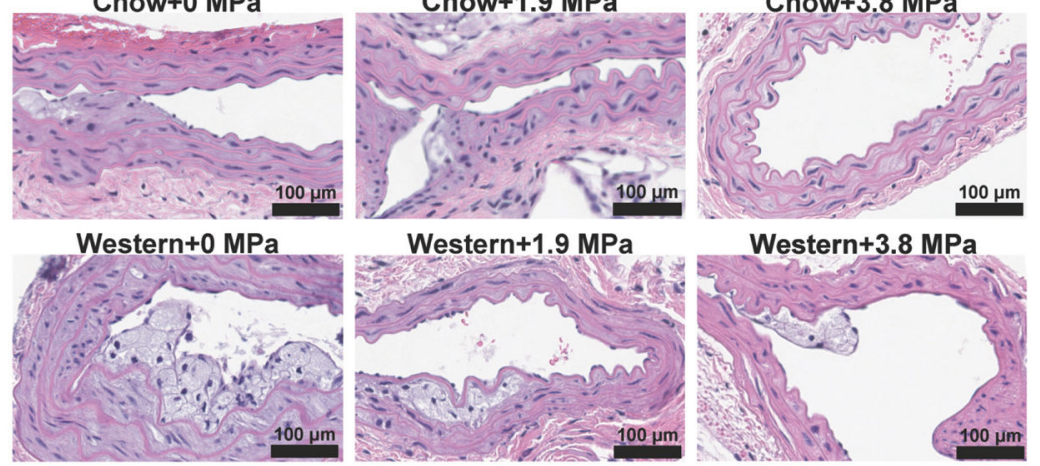

Western+3.8 $\mathrm{MPa}$
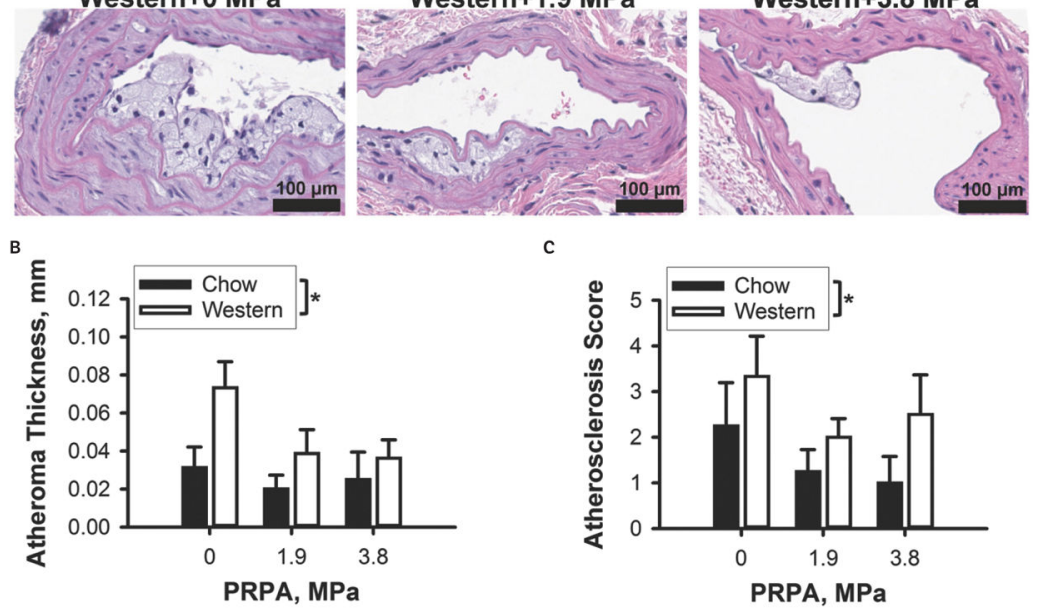

Figure 3.

Aortic histologic analysis. The aortic arch and descending aorta were fixed briefly in situ with 10\% NBF and then carefully excised and fixed in 10\% NBF for an additional 24 hours, embedded in paraffin, sectioned, and stained with hematoxylin-eosin (A). A pathologist blinded to the exposure conditions measured atheroma thickness with an ocular micrometer (B) and assigned an atherosclerosis score between 0 and 5 using the American Heart Association classification scheme for human atherosclerotic lesions (C). Bars indicate SEM $(\mathrm{n}=3-4$ per group $; * P<.05)$. 